\title{
Influence of Precursors of Alkyl Self Assembled Monolayers on the Alkyl Chain Structure Studied by sum Frequency Generation
}

\author{
A.B. EL Basaty ${ }^{1,2}$, Y.Miyauchi ${ }^{1}$, H. Nakayama ${ }^{1}$ H. Sano ${ }^{3}$, \\ and $\mathbf{G}$ Mizutani ${ }^{1}$ \\ School of Materials Science, Japan Advanced institute of Science \\ and Technology \\ 1-1 Asahidai, Nomi, Ishikawa, 923-1292, Japan ${ }^{1}$ \\ Physics Department, Faculty of Science, Helwan University, Headquarter, Ain \\ Helwan, Helwan governorate, Egypt, ${ }^{2}$ \\ Ishikawa National College of Technology, Kitachujo, Tsubata, Kahoku-gun, \\ Ishikawa, 929-0392 Japan ${ }^{3}$ \\ mizutani@jaist.ac.jp,TEL.+81-0761-51-1521, FAX. +81-761-51-1149
}

We have performed infrared visible sum frequency generation (SFG) spectroscopy of alkyl silane SAMs on quartz substrates prepared by chemical vapor deposition (CVD) of Octyldimethylmethoxysilane (ODMS) and Octyltrichlorosilane (OTCS). ODMS and OTCS have eight carbon $\left(\mathrm{C}_{8}\right)$ chains with one and three precursors, respectively. The dependence of the structure of the alkyl chains on the precursor numbers was investigated. In the SF spectra, symmetric and asymmetric methylene stretching vibrations were observed at 2849 2852 and 2910 2918 $\mathrm{cm}^{-1}$, respectively. The strongest peak at 2876 2879 $\mathrm{cm}^{-1}$ was attributed to the symmetric stretch of methyl end group. The peak intensity ratios of methylene stretching vibrations to symmetric methyl vibration of the ODMS monolayer were larger than that of OTCS. This result indicates that the number of gauche defects was reduced with the increase of the number of precursors. In the ODMS SAMs with one precursor the molecules are packed with low density due to the unstable anchoring and thus the van der Waals interaction between alkyl chains is weak. This should lead to the increased number of the gauche defects in the ODMS SAMs. 


\section{Introduction:}

Self assembled monolayers (SAMs) are studied extensively, since the monomolecular layer on metallic, semiconductor and dielectric surfaces modifies the surface properties dramatically in wetting, adhesion, lubrication, or corrosion.[1,2,3] Moreover, they were applied to nanotechnology and biology such as anchoring and immobilizing a protein and a DNA [4]. In spite of the vigorous studies, the packing dynamics of SAMs is still unclear, since it depends on many factors in an extremely complicated way. As typical well defined SAMs, alkylsilane SAMs on quartz or Si surfaces have been studied extensively. In these studies the most important factor is presumably the alkyl chain length. Due to the van der Waals interaction between the alkyl chains within the monolayer, SAMs with longer chain length like $\mathrm{C}_{18}$ chains are ordered and packed very well. On the other hand, SAMs with chains shorter than $\mathrm{C} 10$ are more flexible, [5] and have rather lower order. [6].

The number and symmetry of the precursors are also important for the packing. For instance, octadecyltrichlorosilane (OTS) with $\mathrm{C}_{18}$ chains have three chlorine atoms in the head groups and they are precursors. On a quartz surface, one precursor of an OTS molecule anchors to the surface, while the others form Si-O-Si cross linkage.[6]. Due to the siloxane network, the OTS molecules are well assembled with high packing density. [7].

Recently, Lee et al. reported the influence of the molecular symmetry of the precursor on the orientation and the packing density of the SAMs. [8]. They carried out FT-IR spectroscopy of different alkylsilane SAMs with one to three precursors. In their detection, the silane molecules with three hydroxyl head groups stood upright on a Si surface, and grew up to a monolayer with high molecular density. When the number of the precursors was decreased, the molecular density in the SAMs decreased. Even though their measurements and modeling were excellent, they did not discuss the alkyl chain structure. In order to understand the packing dynamics of SAMs, one has to investigate how the alkyl chain structure changes with the number of precursors, since the packing of the high flexible chains is considered to be the key point.

In this study, we have performed vibrational sum frequency generation (SFG) spectroscopy of alkylsilanes on quartz substrates, and compared the spectra between different numbers of precursors. SFG is a well established technique for analyzing alkyl chain structures of SAMs on solid surfaces. $[9,10]$ We used two different alkylsilanes having $\mathrm{C}_{8}$ chains, Octyldimethylmethoxysilane (ODMS) and Octyltrichlorosilane (OTCS) with one $\mathrm{OCH}_{3}$ precursor and three $\mathrm{Cl}$ precursors, respectively. The chain length of 
the molecules studied in this work is much shorter than that of $\mathrm{C}_{12}$ or $\mathrm{C}_{18}$ chains in previous reports. $[2,10]$. We chose the SAMs with $\mathrm{C}_{8}$ chains because the weaker interaction between shorter chains should allow the flexibility of the chain structures and increase the influence of the number of the precursors. $[5,11]$.

\section{Sum Frequency Spectroscopy:}

Vibrational SFG is a second order nonlinear optical process excited by a mid-IR pulse tuned near a molecular vibrational resonance and a nonresonant visible pulse. The SFG intensity depends on the second order susceptibility, $\chi^{(2)}$. Here $\chi^{(2)}$ is a third rank tensor and depends on the average orientation of the surface molecules and the coverage. The SFG intensity $I_{S F G}$ and the intensity of the visible and infrared light $I_{v i s}$ and $I_{I R}$ are related as,

$$
I_{S F G} \propto\left|\chi^{(2)}\right|^{2} I_{\text {vis }} I_{I R}
$$

In the dipole approximation, all elements of $\chi^{(2)}$ vanish in centrosymmetric media, but they are nonzero at surfaces and interfaces, since the structure of the surfaces and interfaces does not have a center of inversion. The scalar component of the second order susceptibility, $\chi^{(2)}$ can be written as

$$
\begin{aligned}
& \chi^{(2)}=\sum_{n}\left|\chi_{R, n}^{(2)}\right| e^{-i \Phi_{n}}+\chi_{N R}^{(2)} \\
& \chi_{\mathrm{R}, \mathrm{n}}^{(2)}=\frac{\mathbf{A}_{\mathrm{n}}}{\omega_{\mathrm{IR}}-\boldsymbol{\omega}_{\square}+\mathrm{i}_{\mathrm{n}}}
\end{aligned}
$$

where $\chi_{R, n}^{(2)} \chi_{N R}^{(2)}, \quad \Phi_{n}, A_{n}, \omega_{\mathrm{n}}$, and $\mathrm{r}_{\mathrm{n}}$ are a resonant second order nonlinear susceptibility component, a non-resonant component, a phase angle between the resonant and non-resonant contributions, the amplitude, frequency and a damping constant of the $n$th surface vibrational mode, respectively. SFG spectroscopy gives vibrational spectra of noncentrosymmetric molecules. The information is different from that offered by IR and Raman vibrational spectroscopes. [12, 13]. 


\section{Experiment :}

\subsection{Sample preparation:}

In this study, SAMs of alkylsilanes (ODMS and OTCS) were deposited on hydrophilic surfaces of quartz substrates (DAICO MFG Co.) by a chemical vapor deposition (CVD) method [17]. In the pre-treatment, we dipped the quartz substrates in acetone aqueous solution and then in pure water under ultrasonic treatment for 10 minutes, in order to remove organic impurities on the surfaces. These treatments were repeated twice. Then we dried the substrates in a dry oven, and then irradiated them with UV light for 1 hour. Octyldimethylmethoxysilane (ODMS; $\mathrm{CH}_{3}\left(\mathrm{CH}_{2}\right)_{7}\left(\mathrm{CH}_{3}\right)_{2} \mathrm{Si}\left(\mathrm{OCH}_{3}\right)$ and Octyltrichlorosilane (OTCS; $\left.\mathrm{CH}_{3}\left(\mathrm{CH}_{2}\right)_{7} \mathrm{SiCl}_{3}\right)$ were used as received from Shin-Etsu Chemicals Co. Ltd. The substrate and a small plastic cup of $0.2 \mathrm{~mL}$ of alkylsilane were put together in a Teflon cup in $\mathrm{N}_{2}$ environment, and then the Teflon cup was sealed with a cap. The cup was placed in an electric oven. The temperature of the oven was raised from room temperature to $373 \mathrm{~K}$, and was maintained at $373 \mathrm{~K}$ for 4 hours. After that, the substrates were taken out of the cup quickly and were immediately dipped in distilled water and then in an isopropyl alcohol (IPA) aqueous solution for 10 seconds. This dipping in two kinds of liquids was repeated twice. Finally, the substrates were dried at $120^{\circ} \mathrm{C}$ for 2 minutes.

The quality of the fabricated SAMs was characterized by a contact angle (CA) method with pure water droplets of $\approx 5 \mu \mathrm{L}$ each. ${ }^{2}$ The CA of OTCS SAMs was $\approx 95 \pm 4^{\circ}$ and that of ODMS was $\approx 82 \pm 1^{\circ} . .^{15}$ The CA $\approx 95 \pm 4^{\circ}$ of OTCS indicates that the SAMs are a well ordered monolayer. The CA $\approx 82 \pm 1^{\circ}$ of ODMS was larger than that of Octadecyldimethylchlorosilane with one precursor reported by Lee et al. Thus, the ODMS molecules are judged to cover the quartz substrate surface very well. [8]

Surface roughness of the substrate was reported to induce disorder in the orientation of molecules in SAMs. [16]. Hence we measured the surface roughness of our substrate by using atomic force microscopy (AFM). The average substrate roughness amplitude was $\mathrm{Rms} \approx 0.38 \mathrm{~nm}$ and the average tilt angle was $\approx 0.06^{\circ}$. This observation indicates that the substrate surface was too smooth to influence the packing of the SAMs.

\subsection{SFG measurement}

The experimental setup for the SFG spectroscopy has been reported elsewhere [17, 18]. Doubled frequency output at the wavelength of $532 \mathrm{~nm}$ from a mode-locked $\mathrm{Nd}^{3+}$ :YAG laser and the tunable IR light with wavenumber from 2800 to $3000 \mathrm{~cm}^{-1}$ from OPG/OPA-DFG were irradiated on the sample. 
The incident angles of the visible and IR light beams were $\sim 70^{\circ}$ and $\sim 40^{\circ}$, respectively. The pulse energies of visible and IR beams were 31.7 and $105.9 \mu \mathrm{J} /$ pulse, respectively. The polarization of SFG, visible and IR light were $s, s$ and $p$, respectively. This polarization combination ( $s s p)$ is sensitive only to the vibrational mode with the atomic displacement normal to the surface. ${ }^{12}$ SF intensity of the samples were normalized by that from a GaAs(001) reference. The SFG measurements were carried out at room temperature and ambient conditions.

\section{Results and Discussion:}

The spectrum (a) in Fig. (1) shows a SFG spectrum of OTCS monolayer on a quartz substrate. In the spectrum, the peak at $2876 \mathrm{~cm}^{-1}$ is attributed to the symmetric stretch $\left(\mathbf{r}^{+}\right)$of methyl end group, and the peak at $2945 \mathrm{~cm}^{-1}$ is assigned to Fermi resonance $\left(\mathbf{r}_{\mathrm{FR}}^{+}\right)$of $\mathbf{r}^{+}$mode and the overtone of a $\mathrm{CH}_{3}$ bending mode. The remaining peaks at $2849 \mathrm{~cm}^{-1}$ and $2918 \mathrm{~cm}^{-1}$ are attributed to methylene symmetric $\left(\mathbf{d}^{+}\right)$and asymmetric $\left(\mathbf{d}^{-}\right)$stretching modes. [12, 19, 20]. The peak of $\mathrm{r}^{+}$mode is the highest in the spectrum. The ratio of intensities of $\mathbf{d}^{+}, \mathbf{d}^{-}$, and $\mathbf{r}_{\mathrm{FR}}^{+}$modes to $\mathbf{r}^{+}$mode are $47 \%, 41 \%$, and $98 \%$, respectively. The spectrum of ODMS monolayer is shown in the spectrum (b) in Fig. (1). The $\mathbf{r}^{+}, \mathbf{d}^{+}$, and $\mathbf{d}^{-}$modes are observed at $2879,2852 \mathrm{~cm}^{-1}$, and $2910 \mathrm{~cm}^{-1}$, respectively. ${ }^{12,21,22,23}$ The peak at $2950 \mathrm{~cm}^{-1}$ is assigned to $\mathbf{r}_{\mathrm{FR}}^{+}$mode or out of plane $\mathrm{C}-\mathrm{H}$ asymmetric stretch $\left(\mathbf{r}^{-}\right)$modes of the terminal methyl $\left(\mathrm{CH}_{3}\right)$ group. $[22,24]$.

The ratios of intensities of $\mathbf{d}^{+}$and $\mathbf{d}^{-}$modes to $\mathbf{r}^{+}$mode are $69 \%$ and $72 \%$, respectively, in the spectrum (b) in Fig. 1. Obviously, the ratios of $\mathbf{d}^{+}$ and $\mathbf{d}^{-}$modes in the spectrum (b) are higher than those of the corresponding modes in the spectrum (a). From a general discussion, ${ }^{12,}{ }^{20}$ the higher ratios in Fig. ( 1 b) indicate more gauche defects in the alkyl chains of ODMS monolayer. In all-trans SAMs configuration, the structure of the methylene groups in the alkyl chains are considered to be nearly centrosymmetric. Thus, the SFG signals attributed to $\mathbf{d}^{+}$and $\mathbf{d}^{-}$modes from alkyl SAMs with all-trans methylene groups have to be small. ${ }^{20}$ Thus the small ratios of $\mathbf{d}^{+}$and $\mathbf{d}^{-}$modes to $\mathbf{r}^{+}$mode in spectrum (a) in Fig. 1 represent that the alkyl chains of OTCS monolayer is nearly all-trans. On the other hands, the structure of $\mathrm{CH}_{2}$ pairs in the gauche configuration of alkyl chains lacks the inversion symmetry. The intensities of $\mathbf{d}^{+}$and $\mathbf{d}^{-}$modes are correlated with the number of defects in the alkyl chain. [19]. Thus, the high ratios of $\mathbf{d}^{+}$and $\mathbf{d}^{-}$modes in the spectrum (b) indicate the larger number of gauche defects in the monolayer. 


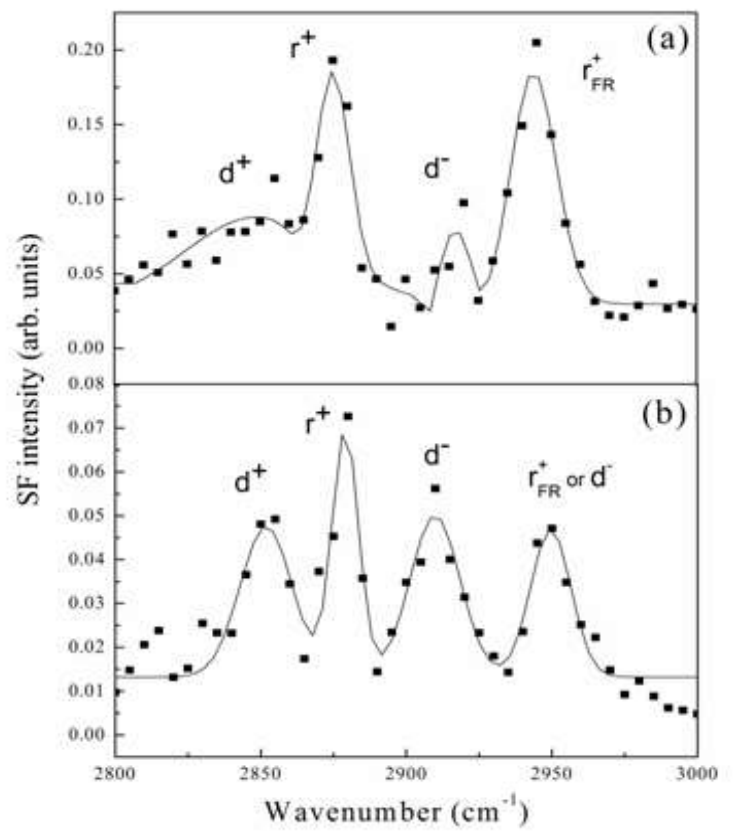

Fig.(1): SFG spectra of (a) Octyltrichorosilane (OTCS) SAMs and (b) Octyldimethylmethoxysilane (ODMS) SAMs on a quartz substrate, the solid curves are the Gaussian fitting of the peaks

Figure (2) depicts a schematic diagram of the conformation of the alkysilane monolayers. According to the report by Lee et al., the chlorine atoms in the head groups of Octadecyltrichlorosilane (OTS) are displaced with the hydroxyl groups maintaining the three-fold symmetry and then one head group get chemically bonded to a Si substrate. [12] Due to this symmetry property the OTS molecules can stand upright with the siloxane network. Similarly, the OTCS molecules should stand upright and construct siloxane network. Accordingly the OTCS monolayer should have high packing density. This argument is consistent with the observed high contact angle $\sim 95^{\circ}$ of the OTCS monolayer. Due to the strong van der Waals interaction between the alkyl chains in the packing, nearly all-trans alkyl chains are maintained.

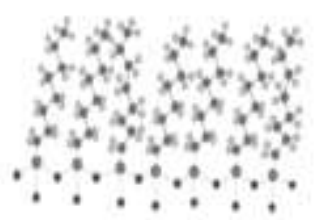

(a) OTCS

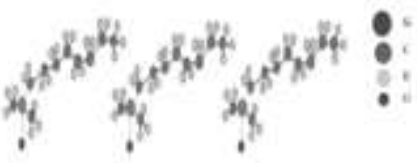

(b) ODMS

Fig.(2): A schematic diagram of the structures of (a) OTCS and (b) ODMS monolayers. 
On the other hand, the lower contact angle $\sim 82^{\circ}$ of ODMS monolayer indicates its lower packing density. The ODMS molecule has only one hydroxyl head group before bonding to the substrate. Thus, due to the high flexibility of the chains and lack of connectors for cross linkage, the molecules lay down on the substrate and the free volume in the SAMs plane increases. The lower density of the alkyl chains reduces the van der Waals interaction, and thus induces gauche defects.

This study not only confirmed that the SAMs chains lay down with the decrease of the number of the precursors as reported in the study by FT-IR and AFM measurements, ${ }^{8}$ but also found the increase of gauche defects with the decrease of the number of the precursors.

\section{Conclusions:}

In summary, we compared SFG spectra of ODMS and OTCS SAMs on quartz substrates. The number of gauche defects in ODMS SAMs was found to be higher than that in OTCS SAMs. We have observed change of alkyl chain structure of alkylsilane SAMs as a function of the number of precursors.

\section{References:}

1. K. Cimatu, and S. Baldelli, J. Phys. Chem. B 110, 1807 (2006).

2. S. A. Kulkarni, K. P. Vijayamohanan, J. Surface Science, 601, 2983 (2007).

3. Z. Guo, W. Zheng, H. Hamoudi, C. Dablemontb, V.A. Esaulovb, and B. Bourguignona, Surface Science, 602, 3551 (2008).

4. S. Sun, M. Montague, K. Critchley, M. S. Chen, W. J. Dressick, and G. J. Leggett, NANO LETTERS, 6, 29 (2006).

5. M. D. Porter, T. B. Bright, D. L. Allara, and E. D. Chidsey, J. Am. Chem. Soc. 109, 3559 (1987)

6. H. Yamamoto, T. Watanabe, and I. Ohdomari, J of Chemical Physics 128, 164 (2008).

7. D. L. Angst, and G. W. Simmons, Langmuir, 7, 2236 (1991).

8. S. H. Lee, N. Saito, and O. Takai, Jpn. J. Appl. Phys. 46, 1118 (2007).

9. H. Asanuma, H. Noguchi, Y. Huang, K. Uosaki, and H. Z. Yu, J. Phys. Chem. C, 113, 21139 (2009).

10. N. Nishi, D. Hobara, M. Yamamoto, and T. Kakiuchi, J. Chem.Phys. 118, 1904 (2003).

11. P. B. Miranda, V. Pflumio, H. Saijo, and Y. R. Shen, Thin Solid Films, 327329, 161-165 (1998). 
12. Y. R. Shen, S. Nihonyanagi, and K. Uosaki, Phys. Chem. Chem. Phys. 3, 3463 (2001).

13. Y. R. Shen, the Principles of Nonlinear Optics, Wiley, New York (1984).

14. T. Koga, M. Morita, and H. Ishida, Langmuir 21, 905 (2005).

15. Y. Yang, A. M. Bittner, S. Baldeli, and K. Kern, Thing Solid Film, 516, 3948 (2008).

16. S. D. Mor'e, H. Graaf, M. Baune, C. Wang, and T. Urisu, Jpn. J. Appl. Phys. 41, 4390 (2002).

17. G. Mizutani, T. Koyama, S. Tomizawa, H. Sano, Spectrochimica Acta Part A 62, 845 (2005).

18. H. Sano, H. Yoshida, T. Oosugi, T. Murakami, Y. Takagawa, G. Mizutani, T. Ooya, and N. Yui, Surface Science, 601, 5173 (2007).

19. P. Guyot-Sionnest, J. H. Hunt, and Y. R. Shen, Phys. Rev. Lett. 59, 1597 (1987).

20. A. S. Lagutchev, K. J. Song, J. Y. Huang, P. K. Yang, and T. J. Chuang, J. Chemical Physics, 226, 337 (1998).

21. M. Himmelhus, F. Eisert, M. Buck, and M. Grunze, J. Phys. Chem. B 104, 576 (2000).

22. R. C. Chow, T. T. Ehler, and T. E. Furtak, Appl. Phys. B 74, 395 (2002).

23. S. Ma, M. Ano, and T. Suzuki, Collids and surfaces. Eng. Aspects, 257258, 357-362 (2005).

24. K. Cimatu, H. Justin Moore, Barriet, P. Chinwangso, T. Randall Lee, and S. Baldelli, J. Phys. Chem. C 112, 14529 (2008). 